EPJ manuscript No.

(will be inserted by the editor)

\title{
Optimal disorder for segregation in annealed small worlds
}

\author{
Santiago Gil and Damián H. Zanette ${ }^{a}$ \\ Centro Atómico Bariloche and Instituto Balseiro, 8400 Bariloche, Río Negro, Argentina \\ Received: date / Revised version: date
}

\begin{abstract}
We study a model for microscopic segregation in a homogeneous system of particles moving on a one-dimensional lattice. Particles tend to separate from each other, and evolution ceases when at least one empty site is found between any two particles. Motion is a mixture of diffusion to nearest-neighbour sites and long-range jumps, known as annealed small-world propagation. The long-range jump probability plays the role of the small-world disorder. We show that there is an optimal value of this probability, for which the segregation process is fastest. Moreover, above a critical probability, the time needed to reach a fully segregated state diverges for asymptotically large systems. These special values of the long-range jump probability depend crucially on the particle density. Our system is a novel example of the rare dynamical processes with critical behaviour at a finite value of the small-world disorder.
\end{abstract}

PACS. 89.75.Fb Structures and organization in complex systems - 02.50.Ey Stochastic processes

Segregation -the spatial separation of different elements in a multi-component system- is a paradigmatic form of complex behaviour, present in a wide variety of natural phenomena. It can occur both at the microscopic ' level, driven by repulsive interactions between individual components, and at the macroscopic level, as in the case of phase separation due to collective processes. In many instances, it leads to the spontaneous emergence of spatiotemporal order, in the form of coherent structures with non-trivial evolution.

In physical systems, spatial segregation is conspicuous in the vicinity of phase transitions, as the result of the amplification of microscopic fluctuations 11. An effect of similar origin is observed in some elementary chemical reactions limited by diffusion. It is well known, for example, that the binary annihilation of two diffusing chemicals $A$ and $B, A+B \rightarrow 0$, leads to the formation of single-species spatial domains just after the very first stages of the process, starting from a uniform mixture of the two species [2].

In the realm of biology, segregation at the molecular and cellular level plays a prominent role in morphogenesis, i.e. in the differentiation of the various tissues, organs, and functional parts of living organisms $[\underline{3}$. At the level of ecosystems, environmental heterogeneities may lead to population segregation, with the appearance of specific localized niches, when the relative fitness of competing species varies in space 4]. In human populations, the combination of cultural diversity with xenophobic feelings is able to disrupt society, generating hatred and aggression where cooperative behaviour should otherwise emerge [5].

\footnotetext{
a Also at Consejo Nacional de Investigaciones Científicas y Técnicas, Argentina.
}

Homogeneous ecological and social systems, with no substantial differences between their individuals, often exhibit a different form of segregation, not necessarily associated with the formation of spatial structures. Single individuals -or small groups of closely related individuals, such as families or clans- may choose to separate from each other as much as possible, all over the available spatial domain. This spreading is related to a more efficient exploitation of the resources associated with land, and has certainly been crucial to the geographical dissemination of biological species over the ages [4].

In this paper, we explore a physically-inspired model for this kind of segregation. We pay particular attention to the dynamical process that leads, from a random distribution of the population, to a stationary state where individuals have mutually separated such that no contiguous neighbours are found. Individuals are allowed to move diffusively and, occasionally, they can perform long jumps to randomly selected sites. This form of transport, inspired in the connection structure of small-world networks, is discussed in the next section. In Sect. 2] we present our first numerical results, and show that the time needed to reach full segregation depends non-monotonically on the probability of long jumps. Remarkably, in fact, the time is minimal for an intermediate value of that probability. Section [3 is devoted to develop an analytical approximation of our problem, which makes it possible to explain some of the numerical results. Further simulations are presented in Sect. 4. where we validate predictions of the analytical approximation and complete the study of our system. Results are summarized and discussed in the last section. 


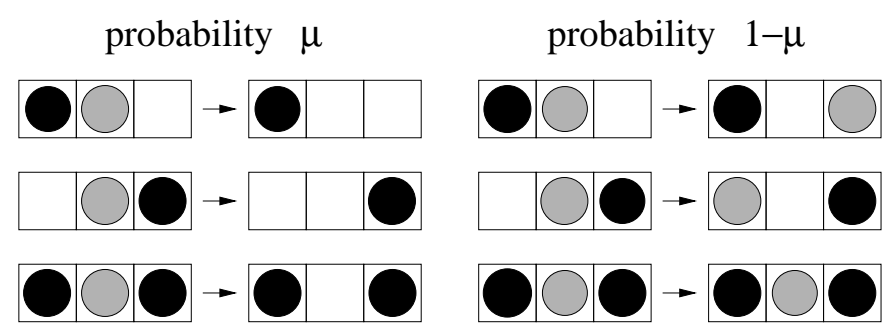

Fig. 1. Possible events associated with the motion of a frustrated particle. The moving particles is shown in gray. Left column: long-range jump events. Right column: diffusion events.

\section{Segregation in annealed small worlds}

We consider an ensemble of $N$ particles distributed on a one-dimensional array of $L$ sites $(L \geq N)$ with periodic boundary conditions. The density is $\rho=N / L$. An exclusion principle holds so that, at any time, each site may be occupied by at most one particle. Particles tend to segregate, and a particle is said to be frustrated if at least one of its two nearest-neighbour sites is occupied. Frustrated particles are allowed to move, in order to reach a non-frustrated state. Motion of frustrated particles is governed by annealed small-world transport, as described in the following.

Small-world networks have been defined to capture two essential properties of real social structures, namely, the small average distance between any two individuals (small-world effect), and their high clustering 6. They have been used, as toy models, to demonstrate the role of the underlying interaction pattern in dynamical processes of socio-economical and ecological inspiration 7. 8. 9. These networks are built starting from an ordered lattice of relatively high connectivity. A prescribed fraction of links is reconnected at random, thus introducing quenched disorder and creating shortcuts between distant parts of the original lattice. Annealed small worlds constitute a variation of the same model, introduced for systems where propagation processes occur between sites [10,11, 9. In this version of the small-world model, propagation takes place between neighbour sites of the ordered lattice but, with a certain probability, jumps to randomly chosen sites may take place. Long-range jumps play the role of the shortcuts of the quenched original version. By analogy, these random jumps are associated with disorder in the propagation process.

In our model of segregation, a particle is chosen at random at each time step. If it is not frustrated, it remains at its site. If, on the other hand, the selected particle is frustrated, it is allowed to jump to a randomly selected empty site, anywhere in the lattice, with probability $\mu$. With the complementary probability, $1-\mu$, the motion is diffusive. If only one of the nearest-neighbour sites is occupied, the particle moves to the empty neighbour. If, otherwise, the two nearest-neighbour sites are occupied, the exclusion principle forbids motion. The possible instances are illustrated in Fig. 10 As discussed above, the parameter $\mu$ can be associated with the level of disorder of the annealed small world. The duration of each time step,
$\Delta t=1 / N$, is fixed so that, on the average, each particle is chosen once per time unit.

We may assert a priori that the system has two welldifferentiated regimes, depending on how the density $\rho$ compares with $1 / 2$. For $\rho \leq 1 / 2$, collective states where no particle is frustrated exist. For finite $L$, moreover, the population will necessarily reach one of such fully nonfrustrated states, from any initial condition, in a finite time $T$. From then on, it will remain in a frozen configuration where all particles are mutually separated by at least one empty site. On the other hand, for $\rho>1 / 2$, there will always be frustrated particles, as there is not enough place to accommodate the population in a fully non-frustrated state. As time elapses, the number of frustrated particles will eventually fluctuate around a well-defined stationary value.

Following this discussion, our analysis of the present model focuses on the study of the evolution of the number of frustrated particles. In the low-density regime, where the population is able to accomplish full segregation, we record the average time $\bar{T}$ needed to reach the fully nonfrustrated state, starting from a random distribution of particles. The average is performed over different realizations of the initial state and of the stochastic evolution. For high densities, on the other hand, we analyze the asymptotic number of frustrated particles. For convenience both in the numerical and in the analytical calculations, instead of recording the number of frustrated particles we follow the evolution of the number $P$ of particle pairs, defined as the number of particles whose nearestneighbour sites to the right are occupied. The time average of the pair density $p=P / N$ acts as an order parameter for the transition between the two regimes. Our main parameters are the particle density $\rho$ and the jump probability $\mu$.

\section{Preliminary numerical results}

We begin by exploring the scaling properties of our model, for fixed $\rho$ and $\mu$, as a function of the system size $L-$ or, equivalently, of the number of particles $N=\rho L$. It results that, for sufficiently large systems, the evolution of the pair density $p$ becomes size-independent when time is suitably scaled by the step duration $\Delta t=1 / N$. In further simulations, we fix $L=5 \times 10^{4}$, for which this asymptotic independence of size already holds.

Figures 2 and 3 show the evolution of the pair density $p$ for several values of the particle density $\rho$ and the two extreme values of small-world disorder, $\mu=0$ and 1 , respectively. Each curve has been obtained by averaging over 100 realizations. For $\mu=0$, as argued above, $p$ vanishes at finite times if $\rho<1 / 2$ and reaches a slightly fluctuating stationary level if $\rho>1 / 2$. The two regimes are separated by the case $\rho=1 / 2$, where $p$ displays a power-law decay with an exponent close to $-1 / 4$.

For $\mu=1$, rather unexpectedly, the transition between the two regimes does not take place at $\rho=1 / 2$ but at a lower density $\rho_{c}$, somewhere between 0.3 and 0.4 . Even when we know that, for $\rho_{c}<\rho<1 / 2$, fully non-frustrated 


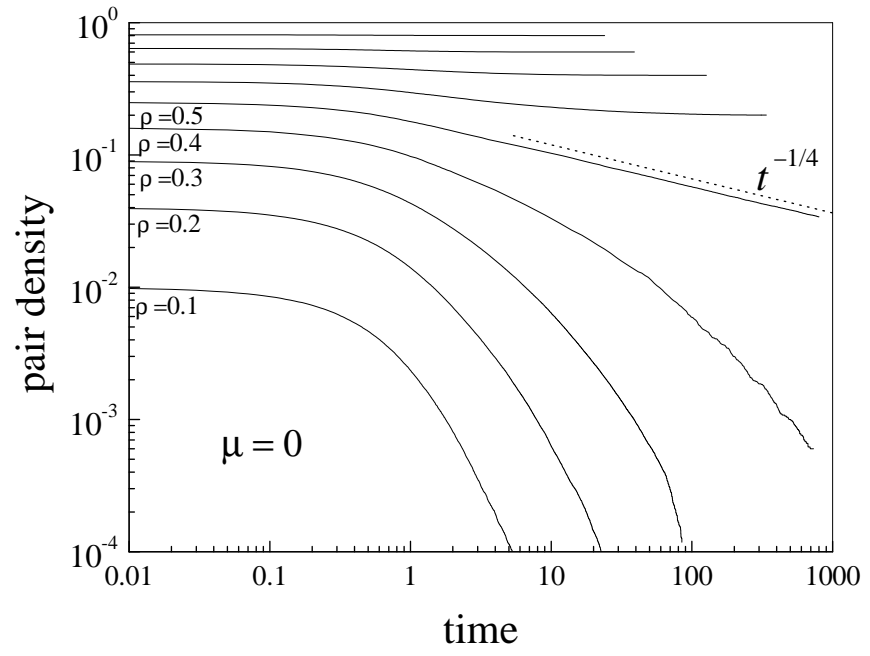

Fig. 2. Evolution of the pair density $p$ for $\mu=0$ and several values of the particle density $\rho$. Curves without labels correspond, from bottom to top, to $\rho=0.6,0.7,0.8$, and 0.9 . The dotted line has slope $-1 / 4$.

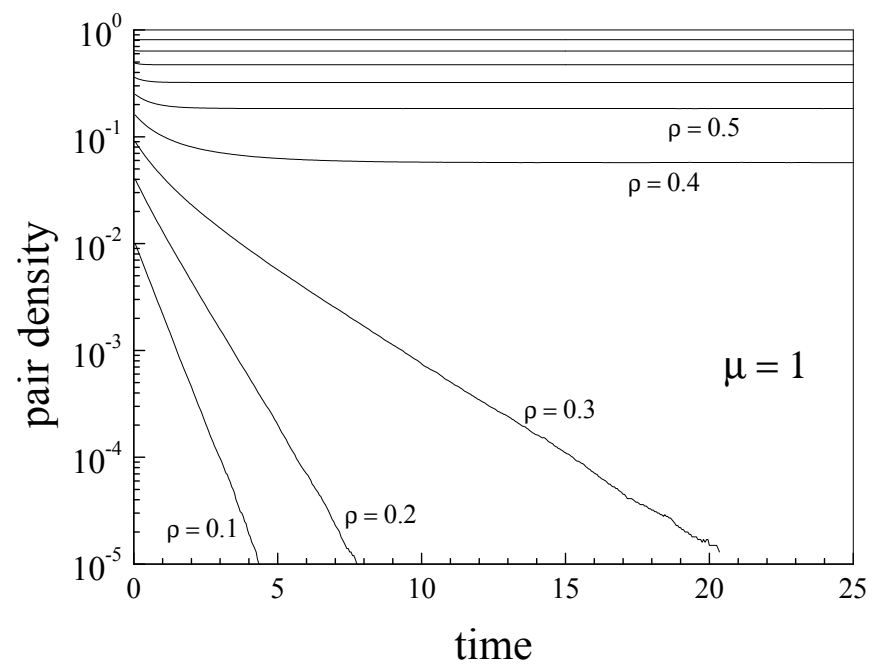

Fig. 3. Evolution of the pair density $p$ for $\mu=1$ and several values of the particle density $\rho$. Curves without labels correspond, from bottom to top, to $\rho=0.6,0.7,0.8$, and 0.9 .

states certainly exist, the system is not able to find any of them and the pair density approaches a stationary nonzero level. It must be recalled that, as discussed above, a finite population will always reach a fully non-frustrated state due to the effect of fluctuations. For the system size of our simulations, however, it is apparent from Fig. 3 that the associated time grows beyond our numerical reach as soon as $\rho$ becomes larger than $\rho_{c}$.

To reveal this feature in more detail and, in particular, to analyze the effect of the small-world disorder, we study the time $T$ needed to reach a fully non-frustrated state. Figure 4 shows the average of $T$, taken over 500 to $10^{4}$

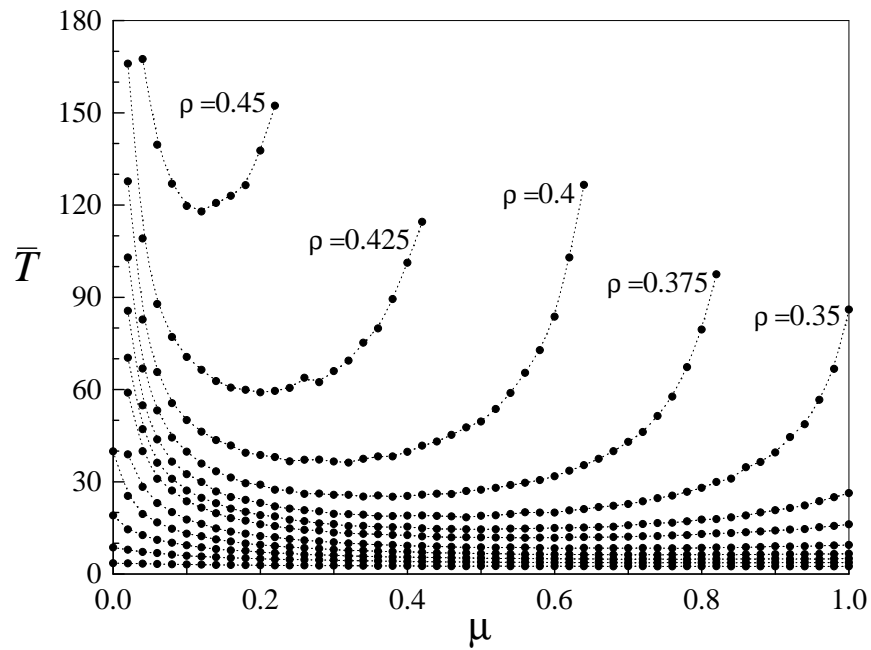

Fig. 4. Average time $\bar{T}$ needed to reach a fully non-frustrated state as a function of the disorder $\mu$, for various values of the density $\rho$. Data without labels correspond, from bottom to top, to $\rho=0.05,0.1,0.15,0.2,0.25,0.3$, and 0.325 . For clarity, data have been joined with dotted lines.

numerical realizations, as a function of the disorder $\mu$ and for several values of the density $\rho<1 / 2$. In agreement with our previous results, we find that, for $\rho \gtrsim 0.35$, there is a critical level of disorder $\mu_{c}$ at which the average time $\bar{T}$ becomes very large. For $\rho \approx 0.35$, we have $\mu_{c} \approx 1$, and the critical disorder decreases as the particle density grows. It seems to approach zero as $\rho$ becomes closer to $1 / 2$.

For fixed $\rho$, the average time $\bar{T}$ grows not only as the disorder increases towards $\mu_{c}$, but also as the disorder decreases and approaches zero. This implies that, remarkably, there is an intermediate value of $\mu$ for which $\bar{T}$ is minimal. This value depends on the density, and decreases as $\rho$ grows. At the same time, the minimum becomes sharper. For small densities, the curve is so flat that the minimum cannot be discerned, due to numerical fluctuations in the value of $\bar{T}$ as $\mu$ varies. For larger densities, on the other hand, the minimum is very well defined. Thus, at least for sufficiently high densities, the segregation process is fastest at an intermediate value of the jump probability. Neither pure diffusion nor random long-range jumps is more efficient, to drive the system to the fully non-frustrated state, than this optimal combination of the two processes.

Before proceeding further with numerical simulations, we turn the attention to the analytical study of our system. Our analytical approach makes it possible to explain -or, at least, to clarify to a certain extent- the numerical result obtained so far. Moreover, it provides a clue to the steps to follow in further simulations. 


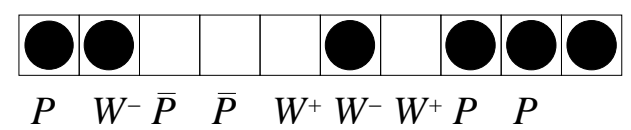

Fig. 5. Pair-antipair representation of a given configuration on a 10 -site domain.

\section{Analytical results}

\subsection{Pair-antipair representation}

We have already introduced particle pairs as an alternative representation of frustrated particles. In this section, we use an extension of this representation as a convenient tool for the analytical treatment of our system.

A particle pair has been assigned to each occupied site whose nearest-neighbour site to the right is also occupied. The number of pairs $P$ is thus given by the number of frustrated particles with a neighbour particle to the right. The density of pairs is $p=P / L$. In the same way, we assign an antipair to each empty site whose nearest-neighbour site to the right is also empty. The number of antipairs is denoted by $\bar{P}$, and their density is $\bar{p}=\bar{P} / L$.

To complete the representation in terms of pair-like elements, we need to introduce the number $W^{-}$of occupied sites whose nearest-neighbours to the right are empty, and the number $W^{+}$of empty sites with occupied sites to the right. However, due to the presence of periodic boundary conditions, we necessarily have $W^{-}=W^{+}$. It is therefore sufficient to consider their sum, $W=W^{-}+W^{+}$, which stands for the total number of "walls" separating pair and antipair domains. The corresponding density is $w=W / L$; we also introduce $q=w / 2=W^{-} / L=W^{+} / L$.

Figure 5 illustrates the pair-antipair representation of a given configuration of the system. Note that, due to the fact that two contiguous elements of this representation share a common site, not all configurations are possible. Specifically, only eight combinations of contiguous elements can occur: $P P, P W^{-}, W^{-} W^{+}, W^{-} \bar{P}, W^{+} P$, $W^{+} W^{-}, \bar{P} W^{+}$, and $\bar{P} \bar{P}$. They correspond to the eight different three-site neighbourhoods in the original representation. All of them are represented in Fig. [5

Expressing the total number of particles and of empty sites in terms of the numbers $P, \bar{P}$, and $W$, it is possible to verify that the densities $p, \bar{p}$, and $q$, and the particle density $\rho$ are related according to

$$
\begin{aligned}
& p+q=\rho, \\
& \bar{p}+q=1-\rho .
\end{aligned}
$$

These relations make it possible to write

$$
\begin{aligned}
& q=\rho-p, \\
& \bar{p}=1+p-2 \rho .
\end{aligned}
$$

Consequently, the only independent quantities in the pairantipair representation are the particle density $\rho$-which, we recall, is one of our parameters- and the pair density $p$. In the frame of this representation, we analyze now the special cases $\mu=0$ and $\mu=1$.
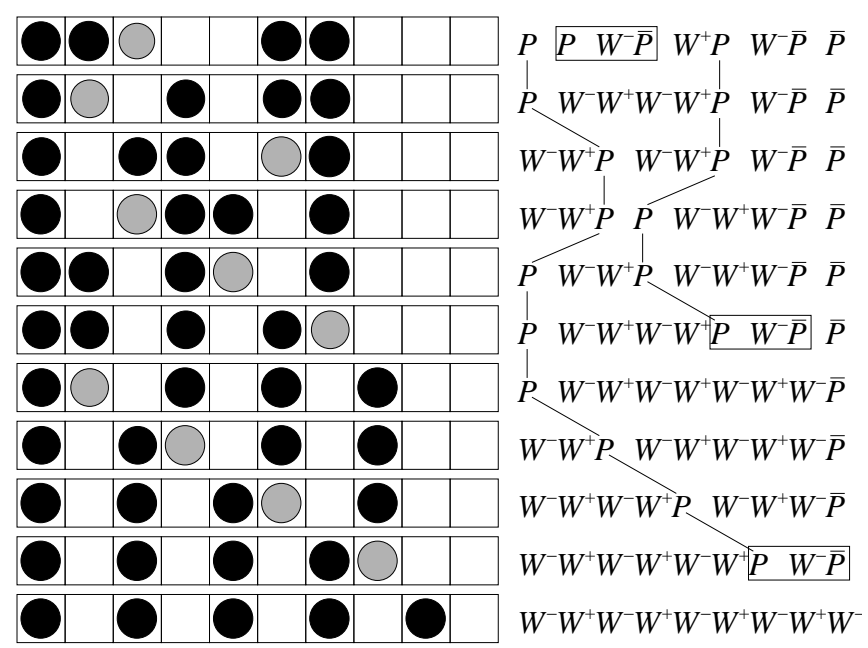

Fig. 6. A realization of the segregation process of five particles, with $\mu=0$ (pure diffusion), within a 10-site spatial interval. Time runs downwards. At each step, the moving particle is shown in gray. To the right, the corresponding pair-antipair representation is given. Boxes indicate pair-antipair annihilation events. Lines stand for pair trajectories.

\subsection{Pure diffusion: $\mu=0$}

For $\mu=0$, frustrated particles can only move to nearestneighbour sites, in the case they are empty, and long-range jumps are not possible. The motion is therefore purely diffusive, with the constraint imposed by the exclusion principle.

Figure [ illustrates a particular realization, during 11 time steps, in a 10-site portion of a larger system. Five particles, all of which are initially frustrated, reach a fully non-frustrated state. To the left of the plot, we show the corresponding (9-site) pair-antipair representation. Pair trajectories are shown with lines, and pair-antipair annihilation events are indicated by boxes.

An exhaustive analysis of the possible configurations in small neighbourhoods reveals that the are three elementary events able to change the configuration. In the pair-antipair representation, they are

$$
\begin{aligned}
& P W^{-} W^{+} \leftrightarrow W^{-} W^{+} P, \\
& P W^{-} \bar{P} \rightarrow W^{-} W^{+} W^{-}, \\
& \bar{P} W^{+} P \rightarrow W^{+} W^{-} W^{+} .
\end{aligned}
$$

The first one can occur in both directions, and stands for the diffusive motion of a pair $P$. Note that $P$ moves two sites to the right or to the left at each step. Antipairs $\bar{P}$, on the other hand, are immobile. The second and third events stand for pair-antipair annihilation. They are equivalent, in the sense that they can be transformed into each other by spatial inversion.

Thus, in the limit of pure diffusion our population is analogous to a reaction-diffusion system of two species, $P$ and $\bar{P}$. While $P$ diffuses through short-range jumps, $\bar{P}$ remains immobile. Moreover, if $P$ and $\bar{P}$ become sufficiently 
close to each other, they may undergo binary annihilation:

$$
P+\bar{P} \rightarrow 0 .
$$

Note that the possibility of this reaction was already implicit in the second of Eqs. (2), which states that the difference $p-\bar{p}$ is a constant of motion (determined by the particle density $\rho$ ).

The analogy with a two-species reaction-diffusion system makes it possible to apply well-know results of diffusion-controlled bimolecular reactions [2]. Specifically, we know that if the densities of pairs and antipairs are identical, $p=\bar{p}$, the kinetics is anomalous. According to the second of Eqs. (2), such condition holds for $\rho=1 / 2$, where the only stationary state for our system corresponds to a periodic array of alternating occupied and empty sites. Under this condition, fluctuations dominate the kinetics 2. Pairs and antipairs segregate into separated spatial domains and, from then on, annihilation takes place at the domain boundaries only. Thus, the kinetics is drastically slowed down and the pair density decreases, for long times, as

$$
p \sim t^{-1 / 4} .
$$

Figure 2 shows that numerical results are in good agreement with this prediction.

For $p \neq \bar{p}$ the approach to stationarity is exponential. If $p<\bar{p}(\rho<1 / 2)$ all pairs are annihilated, while a number of immobile antipairs subsists. Hence, the population is able to reach a fully non-frustrated state. If, on the other hand $p>\bar{p}(\rho>1 / 2)$, moving pairs survive while all antipairs disappear. The remaining density of pairs is $p^{*}=2 \rho-1$, because $\bar{p}=0$. Asymptotically, the population is found in a stationary but dynamical state with a non-vanishing fraction of frustrated particles, as shown in Fig. 2

The analogy with binary annihilation makes it also possible to predict, at least in qualitative terms, the time behaviour of the pair density for $\mu \gtrsim 0$, as compared with the limit $\mu=0$. As long as the disorder remains close to zero, pure diffusion will dominate the transport of particles and, thus, pair and antipair domains will form as a consequence of the mutual annihilation of these two species. While the ensuing annihilation events will mainly occur at the domain boundaries, rare long-range jumps of frustrated particles will give origin to non-local events where distant pairs and antipairs may disappear simultaneously (see also Sect. 3.3). These non-local events take place when a frustrated particle, jumping from a domain occupied by pairs, lands at a antipair domain. Their contribution to the variation of the pair density $p$ is proportional to the product $p \bar{p}$ of the densities of pairs and antipairs, and to the disorder $\mu$. For $\rho=1 / 2(p=\bar{p})$, this contribution implies an algebraic decay $p \sim t^{-1}$; for $\rho \neq 1 / 2$ the decay is exponential. In both cases, it accelerates the decay of the pair density and, thus, the asymptotic state is reached faster. Therefore, the evolution time $T$ must be smaller for $\mu \gtrsim 0$ than for $\mu=0$, as it was shown to be the case in our numerical simulations (Fig. 4).

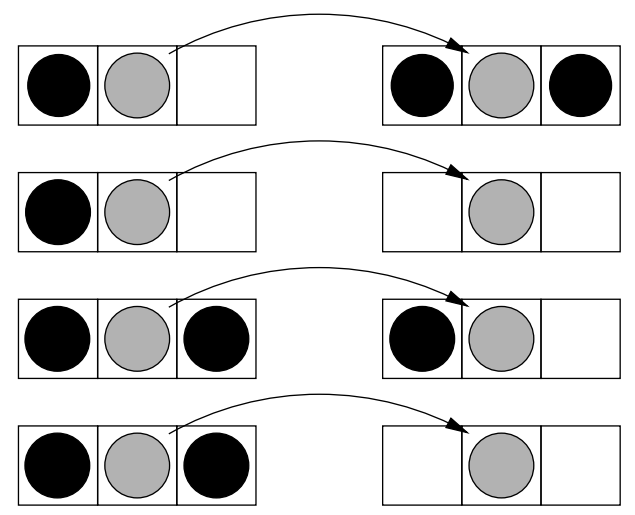

Fig. 7. All the long-range jump events which create or destroy particle pairs (up to spatial-inversion transformations). The moving particle is shown in gray.

\subsection{Full disorder: $\mu=1$}

If $\mu=1$, frustrated particles jump to randomly chosen empty sites, and the small-world disorder is maximal. At each time step, a particle can move a distance of the order of $L$. Regarding the segregation process, however, these random jumps represent an inefficient way of reaching a fully non-frustrated state. As our preliminary numerical simulations have shown, this results into a substantial increase of the times needed to accomplish full segregation.

Since jumps take place to any empty site, it is possible to write an equation for the evolution of the pair density $p$ by evaluating the probability of the events which create or destroy pairs in the limit of an infinitely large system. This approximation requires assuming that the positions of pairs and antipairs are not correlated.

Figure 7 shows -up to the transformation of spatial inversion- all the possible events with net creation or destruction of pairs. The first line displays the only situation where a pair -and the corresponding antipair- can be created. The moving particle starts from a site with one occupied nearest-neighbour $\left(P W^{-}\right)$and lands at the empty site between two occupied sites $\left(W^{-} W^{+}\right)$. The final configuration at the original site is $W^{-} \bar{P}$, and two pairs $(P P)$ have appeared at the landing neighbourhood. We represent this transition as

$$
P W^{-}\left|W^{-} W^{+} \rightarrow W^{-} \bar{P}\right| P P,
$$

where to the left (right) of the arrow we indicate the initial (final) configurations of both the starting and the landing neighbourhoods. The (positive) contribution of this transition to the average variation of the number of pairs $P$ during a time step $\Delta t$ is proportional to the probability of finding the initial neighbourhood configurations $P W^{-}$ and $W^{-} W^{+}$when choosing a particle at random. As for $P W^{-}$, the probability of finding these two elements in contiguous positions -i.e., sharing a particle- is $p q / \rho^{2}$. Equivalently, the probability of finding $W^{-} W^{+}$when choosing an empty site for landing, is $q^{2} /(1-\rho)^{2}$. Thus, the con- 
tribution of the above transition to the variation of $P$ is

$$
\Delta P_{1}=\frac{p q^{3}}{\rho^{2}(1-\rho)^{2}}
$$

Spatial inversion transforms this transition into

$$
W^{+} P\left|W^{-} W^{+} \rightarrow \bar{P} W^{+}\right| P P .
$$

By symmetry, its contribution to the variation of the number of pairs is the same as above, so that $\Delta P_{1}$ will enter the evolution equation for the pair density multiplied by two.

The three last lines in Fig. [7] stand for events where net destruction of pairs - and antipairs- occurs. They correspond to the transitions

$$
\begin{aligned}
& P W^{-}\left|\bar{P} \bar{P} \rightarrow W^{-} \bar{P}\right| W^{+} W^{-}, \\
& P P\left|W^{-} \bar{P} \rightarrow W^{-} W^{+}\right| P W^{-}, \\
& P P\left|\bar{P} \bar{P} \rightarrow W^{-} W^{+}\right| W^{+} W^{-},
\end{aligned}
$$

respectively. In the first two of these transitions only one pair is destroyed, while in the third transition two pairs are simultaneously destroyed. Following the same arguments as above, we can calculate the respective (negative) contributions to the time derivative of the pair density, as

$$
\begin{aligned}
& \Delta P_{2}=-p \bar{p}^{2} q / \rho^{2}(1-\rho)^{2}, \\
& \Delta P_{3}=-p^{2} \bar{p} q / \rho^{2}(1-\rho)^{2}, \\
& \Delta P_{4}=-2 p^{2} \bar{p}^{2} / \rho^{2}(1-\rho)^{2} .
\end{aligned}
$$

The contributions $\Delta P_{2}$ and $\Delta P_{3}$ must be multiplied by two, to take into account the transitions obtained by spatial inversion. On the other hand, the transition giving origin to $\Delta P_{4}$ is invariant under such transformation and, therefore, there is no further contribution associated with it.

In summary, within our approximation, the average variation of the number of pairs $P$ during a time step is given by $\Delta P=2\left(\Delta P_{1}+\Delta P_{2}+\Delta P_{3}\right)+\Delta P_{4}$. The time derivative of the pair density $p=P / L$ can be evaluated as $\dot{p}=\Delta P / L \Delta t=\rho \Delta P$, where we have taken into account that $\Delta t=1 / N$. Using Eqs. (2), (4), and (5), we get

$$
\dot{p}=\frac{2 p}{\rho(1-\rho)}\left(3 \rho^{2}-3 \rho p-\rho+p^{2}\right) .
$$

This equation has three fixed points, corresponding to stationary values of the pair density. They are

$$
p_{0}^{*}=0, \quad p_{ \pm}^{*}=\frac{3}{2} \rho \pm \sqrt{\rho-\frac{3}{4} \rho^{2}} .
$$

Linear stability analysis shows that $p_{+}^{*}$ is unstable for any value of the pair density $\rho$. For $0<\rho<1 / 3, p_{-}^{*}$ is negative and unstable, while $p_{0}^{*}$ is stable. For $\rho>1 / 3$, these two fixed points interchange stability with each other, and $p_{-}^{*}>0$. Therefore, we have a transcritical bifurcation at $\rho_{c}=1 / 3 \approx 0.33$, in good agreement with our numerical results. If the particle density is lower than $\rho_{c}$, the pair density vanishes asymptotically, and the system is able to reach a state where no frustrated particles subsist. For larger particle densities, on the other hand, a fraction $p_{-}^{*}>0$ of the population remains always frustrated. It is remarkable that, according to our prediction, an infinitely large population is unable to find a fully non-frustrated state for $1 / 3<\rho<1 / 2$, even when such states do exist within that density interval. This feature makes clear that too much disorder in transport is inefficient as for the achievement of full segregation.

\subsection{Summary of analytical results}

It is useful to briefly summarize our analytical conclusions, in order to clarify to which extent our previous numerical results have been explained and to define the aims of further simulations. (i) For $\mu=0$, we have established the equivalence between our system and the mutual annihilation of two diffusing species. Known results for the latter make it possible to explain the observed algebraic decay of the pair density, $p \sim t^{-1 / 4}$, for $\rho=1 / 2$ (Fig. 2). We have also shown that, for $\rho>1 / 2$, the asymptotic pair density should be $p^{*}=2 \rho-1$. (ii) For $\mu \gtrsim 0$, it was possible to show qualitatively that the evolution to full segregation is faster than for $\mu=0$, as already found in the simulations (Fig. 4). (iii) For $\mu=1$, we have derived an evolution equation for the pair density $p$. This equation makes it possible to predict, in particular, the asymptotic values of $p$ as a function of $\rho$, from Eq. (7). Also, it predicts a transcritical bifurcation between the regimes of low and high particle densities at $\rho_{c}=1 / 3$. Combining conclusions (ii) and (iii), we can give analytical support to the observation of a minimum in the average time $\bar{T}$ as a function of the disorder $\mu$ (Fig. (4). In fact, while $\bar{T}$ decreases for small $\mu$, it diverges at a finite disorder level, $\mu_{c}<1$.

\section{Further numerical results and phase diagram}

Following our analytical results, it is first pertinent to study in more detail the asymptotic pair density $p^{*}$ for different values of the small-world disorder $\mu$ and of the particle density $\rho$. Figure 8 shows numerical measurements of $p^{*}$ as a function of $\rho$, for four values of $\mu$. For $\mu=0$, results are in excellent agreement with the analytical prediction, $p^{*}=2 \rho-1$, shown as a line. As $\mu$ grows, the dependence of $p^{*}$ on $\rho$ becomes increasingly non-linear and, at the same time, the critical density $\rho_{c}$ shifts to the left. For $\mu=1$, the numerical value of the critical density is slightly above the prediction $\rho_{c}=1 / 3$. Moreover, while analytical and numerical results for $p^{*}$ as a function of $\rho$ are in good agreement, the analytical prediction is systematically larger. As shown in the inset of the fourth panel of Fig. 8] this discrepancy is more important close to the critical point. 

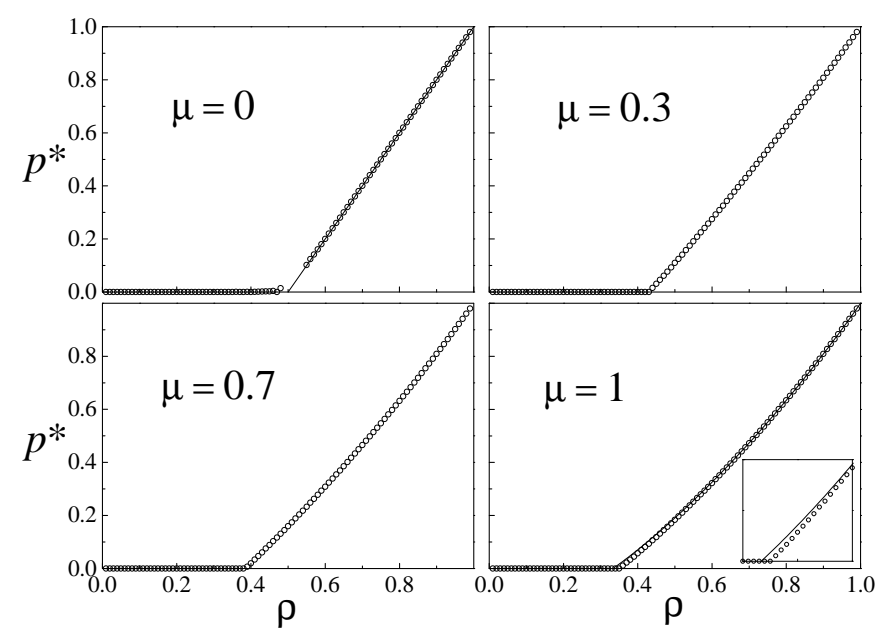

Fig. 8. The stationary pair density $p^{*}$ as a function of the particle density $\rho$, for four values of the small-wolrd disorder $\mu$. For $\mu=0$ and 1 , full lines show the analytical predictions (see Sects. 3.2 and 3.3). For $\mu=1$, the inset shows a close-up of the main plot for $0.3<\rho<0.5$.

As may be expected, the same effect is present in the comparison between the evolution of the pair density for $\mu=1$ as obtained from simulations, and the solution of the evolution equation (6). Figure 9 shows, as full lines, simulation results for various values of the particle density $\rho$, in the same conditions as in Fig. 3 Dotted lines stand the corresponding solutions of Eq. (6). The latter have been obtained through a fourth-order Runge-Kutta integration of the equation with the initial condition $p(0)=\rho^{2}$, which corresponds to a random distribution of particles. The agreement between the two results is very good for small densities, well below the critical value $\rho_{c}=1 / 3$. As $\rho$ approaches $\rho_{c}$, however, a noticeable discrepancy arises in the long-time evolution of the pair density. On the other hand, the initial evolution obtained from simulations is well described by the analytical prediction. The long-time discrepancy seems to reach its largest relative value precisely at the critical density $\rho_{c}$. From then on, its effect decreases steadily, though a difference in the asymptotic values $p^{*}$ persist, as already shown in Fig. 8 .

We identify two possible causes for the discrepancy between simulation and analytical results for $\mu=1$. First, simulations are necessarily affected by finite-size effects. In our system, they convey the possibility that, due to fluctuations, the population reaches a fully non-frustrated state faster than predicted by the average-like arguments of the analytical formulation, which hold for an infinitely large system. We have already stated that, indeed, a finite system should always be able to reach a fully nonfrustrated state in a finite time for any particle density lower than $1 / 2$. Such "early" equilibria may explain the fact that simulation results for the pair density are systematically below the analytical prediction. As expected, the effect is relatively larger close to the critical point.

The second source of discrepancy lies on the uncontrolled assumption made to formulate Eq. (6) that the po-

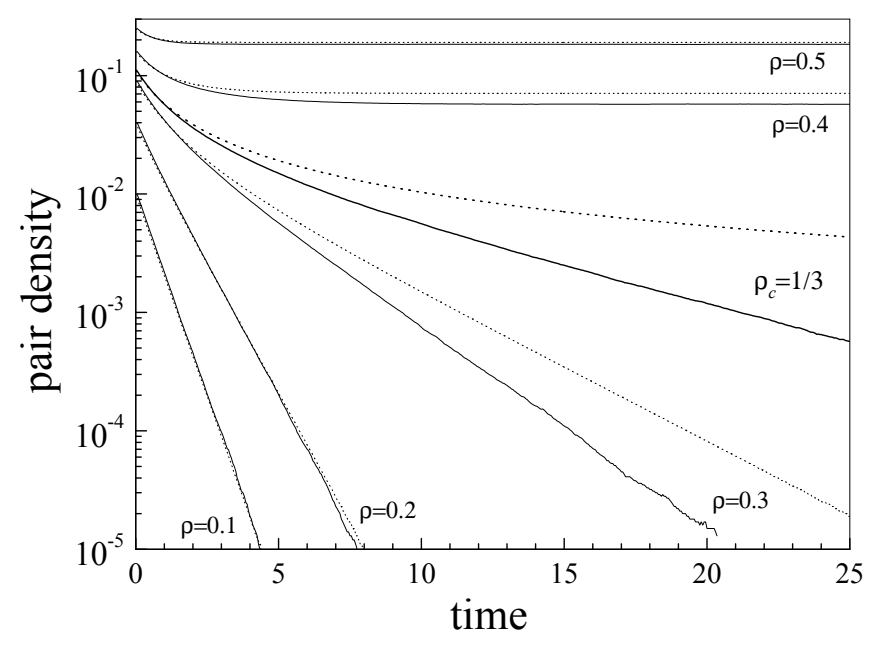

Fig. 9. The evolution of the pair density $p$ for various values of the particle density $\rho$, with $\mu=1$. Full lines correspond to simulation results, and dotted lines stand for the numerical solution of Eq. (6).

sitions of pair-like objects are not correlated. While longrange correlations are surely negligible for the random initial condition, the evolution -which systematically tends to destroy frustrated configurations and to stabilize nonfrustrated states- is expected to create such correlations. This is illustrated in Fig. 6 (for $\mu=0$ ), where the creation of long-range structures such as $\cdots W^{+} W^{-} W^{+} W^{-} \cdots$ is apparent. While the effect of reaching "early" equilibria, discussed above, disappears for $\rho>1 / 2$, the effect of creation of correlations between pair-like objects is present in the whole domain of particle densities.

Our main results for segregation in annealed small worlds are summarized in the phase diagram of Fig. 10] The parameter space $(\mu, \rho)$, spanned by the small-world disorder and the particle density, is divided into two main zones. For low densities, we have the phase where the population is able to reach a fully non-frustrated state while, for large densities, some degree of frustration persists even at asymptotically long times. Within the former, we find the curve over which the average time needed to reach the fully non-frustrated state is minimal. For a given particle density, it gives the optimal small-world disorder to achieve segregation. In the frustrated phase, Fig. [10] shows curves corresponding to constant values for the density of pairs persisting at long times.

\section{Conclusion}

In this article, we have presented and studied a model for microscopic segregation in a homogeneous population of particles moving on a one-dimensional annealed smallworld network. Motion is thus a mixture of short-range diffusion and long-range jumps, picking (frustrated) particles with occupied neighbours and transporting them to empty sites. Small-world disorder is here identified with 


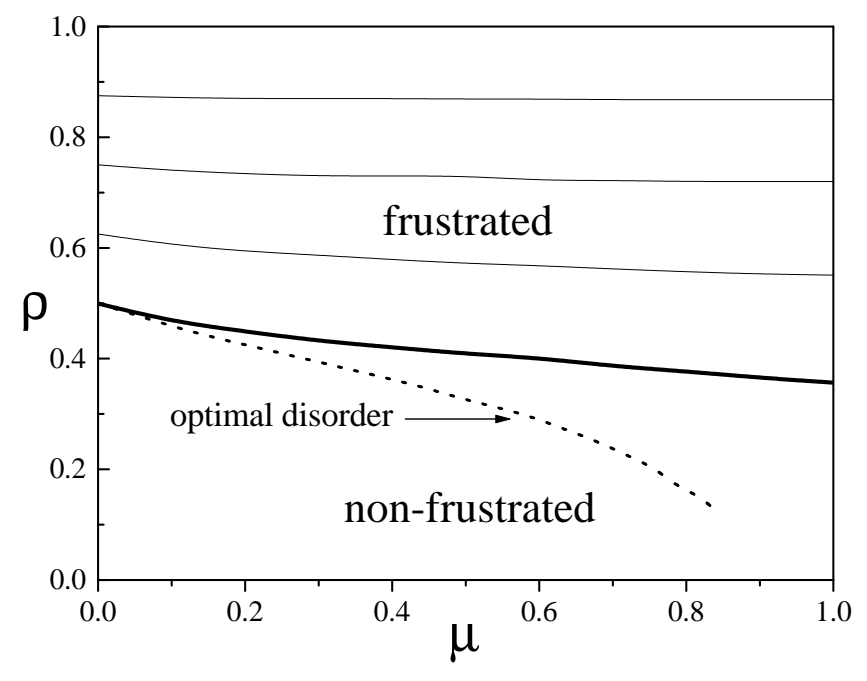

Fig. 10. Phase diagram in parameter space. The bold line separates the region where a fully non-frustrated state is reached from that where frustrated particles subsist for asymptotically long times. The dotted line indicates the values of $(\mu, \rho)$ for which the fully non-frustrated state is reached fastest. Lines in the frustrated phase are level curves of constant stationary pair density $p^{*}$.

the probability of long-range jumps. While no macroscopic separation of phases takes place, for low densities the population is driven toward a (fully non-frustrated) spatial distribution where particles are mutually separated by at least one empty site.

From numerical simulations of the system we have obtained, on the one hand, the time needed to achieve full segregation in the regime of low densities. On the other hand, for high densities, we have analyzed the asymptotic number of persisting frustrated particles. In the parameter space spanned by the small-world disorder and the particle density, we have determined the boundary between the two regimes, which interrelates the two parameters in a non-trivial way. These numerical results have been partially explained by means of an analytical approximation, based on a representation of the particle distribution through pair-like elements. In this representation, the motion of individual particles is associated with events of pair motion or pair-antipair annihilation. The approximation is useful for vanishing small-world disorder, where the segregation process becomes equivalent to the binary annihilation of two diffusing species. In this limit, our approximation makes it possible to predict the anomalous time decay of the number of frustrated pairs at the boundary between the regimes of low and high densities. In the opposite limit of maximal small-world disorder, where particle motion occurs purely through long-range jumps, the approximation predicts a transcritical bifurcation at the boundary. Numerical measurements of the asymptotic density of frustrated pairs are in good agreement with the analytical prediction. Also, the short-time evolution of the pair density is reasonably well approximated by the analytical results.

Our most remarkable results concern the regime of low densities. As stated above, in this regime, the average time needed to reach a fully non-frustrated state is a meaningful overall quantity characterizing the dynamics. We have found, first, that this time attains a minimum at an intermediate value of the small-world disorder. In other words, there is an optimal combination of diffusion and long-range jumps that makes the segregation process fastest. Second, both numerical and analytical evidence show that, in the limit of an infinitely large system, there is a critical value of the small-world disorder above which the time needed to reach the fully non-frustrated state diverges. Too many long-range jumps, thus, make the achievement of full segregation impossible. The critical value of the small-world disorder determines the boundary between the regimes of low and high densities.

Besides the relevance of these conclusions as for the dynamics of segregation itself, they are particularly interesting from the viewpoint of small-world based processes. In fact, it is well known that the threshold of critical behaviour in a large class of systems with underlying smallworld structure shifts toward zero small-world disorder as the system size $N$ grows 12 . This is a direct consequence of the fact that the cross-over from the ordered-like regime to the random-like regime in the geometrical properties of small-world networks occurs at a disorder level of order $N^{-1}$ 13.14. Only a handful of small-world based processes have been reported where critical behaviour or non-monotonic dependence on the disorder persist at finite disorder levels as $N$ is increased 8, 9, 15]. Our system is a novel instance of this rare category, combining both non-monotonic dependence and critical behaviour.

Several generalizations of the present model would be worth considering. First, the same dynamical rules can be implemented in two- or higher-dimensional lattices. In more dimensions, an additional degree of freedom is given by the various ways in which the neighbourhood of a site can be defined. In turn, this determines several possibilities as for the definition of frustrated and non-frustrated states. Another important generalization has to do with the inclusion of noise. In the present version of the model, diffusion events are permitted only when they drive a particle from a frustrated state to a non-frustrated state. In this sense, diffusion in our model is unidirectional toward segregation. Noise can be implemented by allowing a certain degree of reversibility for diffusion events, such that non-frustrated particles might occasionally be lead to frustration. This additional mechanism would be particularly relevant for small disorder levels, when motion is dominated by diffusion. In the pair-antipair representation, reversible diffusion events would be represented by spontaneous creation of a pair and an antipair, $0 \rightarrow P+\bar{P}$, corresponding to the transition $W^{-} W^{+} W^{-} \rightarrow P W^{-} \bar{P}$ or to its spatially inverted form $W^{+} W^{-} W^{+} \rightarrow \bar{P} W^{+} P$. Finally, we recall from the introduction that segregation can be thought of as a process aimed at distributing a population in order to optimize the exploitation of space. 
Our model may be extended to drive the population to a state where the inter-particle distance is maximal, under the constraint imposed by the particle density.

\section{References}

1. D. L. Goodstein, States of Matter (Dover, New York, 1985).

2. V. N. Kuzovkov, E. A. Kotomin, Rep. Prog. Phys. 51, 1479 (1988).

3. J. D. Murray, Mathematical Biology (Springer, Berlin, 1993).

4. P. W. Skelton, Evolution. A Biological and Paleontological Approach (Addison-Wesley, Singapore, 1996).

5. R. Dunbar, Ch. Knight, C. Power, eds., The Evolution of Culture. An Interdisciplinary View (Rutgers University Press, New Brunswick, 1999).

6. D. J. Watts, S. H. Strogatz, Nature 393, 440 (1998).

7. D.J. Watts, Small Worlds (Princeton University Press, Princeton, 1999).

8. M. Kuperman, G. Abramson, Phys. Rev. Lett. 86, 2909 (2001).

9. D. H. Zanette, Phys. Rev. E 65, 041908 (2002).

10. S. C. Manrubia, J. Delgado, B. Luque, Europhys. Lett. 53, 693 (2001).

11. J. Lahtinen, J. Kertész, K. Kaski, Physica A 311, 571 (2002).

12. A. Barrat, M. Weigt, Eur. Phys. J. B 13, 547 (2000).

13. C. F. Moukarzel, Phys. Rev. E 60, R6263 (1999).

14. M. Argollo de Menezes, C. F. Moukarzel, T. F. J. Penna, Europhys. Lett. 50, 574 (2000).

15. A. D. Sánchez, J. D. López, M. A. Rodríguez, Phys. Rev. Lett. 88, 048701 (2002). 